Physics in Collision (PIC 2013)

International Journal of Modern Physics: Conference Series

Vol. 31 (2014) 1460312 (4 pages)

(C) The Authors

DOI: $10.1142 / \mathrm{S} 2010194514603123$

\title{
Energy non-linearity studies at Daya Bay
}

\author{
Masheng Yang* and Yaping Cheng ${ }^{\dagger}$ \\ (On behalf of the Daya Bay Collaboration) \\ Institute of High Energy Physics, Beijing, China \\ *yangms@ihep.ac.cn \\ ${ }^{\dagger}$ chengyp@ihep.ac.cn
}

Published 15 May 2014

\begin{abstract}
The Daya Bay Reactor Neutrino Experiment has measured a non-zero value of the neutrino mixing angle $\theta_{13}$ with a significance of 7.7 standard deviations by a rate-only analysis. ${ }^{1}$ The distortion of neutrino energy spectrum carries additional oscillation information and can improve the sensitivity of $\theta_{13}$ as well as measure neutrino mass splitting $\Delta m_{e e}^{2}$. A rate plus shape analysis is performed and the results have been published. ${ }^{2}$ Understanding detector energy non-linearity response is crucial for the rate plus shape analysis. In this contribution, we present a brief description of energy non-linearity studies at Daya Bay.

Keywords: Neutrino experiment; oscillation; energy response; non-linearity; spectral analysis.
\end{abstract}

\section{General introduction}

It is well established that the flavor of a neutrino oscillates with time. Neutrino oscillations can be described by the three mixing angles $\left(\theta_{12}, \theta_{23}\right.$, and $\theta_{13}$, $)$, a phase of the Pontecorvo-Maki-Nakagawa-Sakata matrix, and the neutrino mass squared differences. $^{3,4}$ To date, the Daya Bay experiments has made the most precise measurement of the neutrino mixing angle $\theta_{13} \cdot{ }^{1,5}$ The Daya Bay Experiment has three underground Experiment Halls $(\mathrm{EH})$ and totally 8 anti-neutrino detectors(ADs). The AD contains a structure of three layers, with Gadolinium loaded Liquid Scintillator(GdLS) in the center, LS in the middle as the gamma catcher, and oil in the outer layer to shield the radioactive components like PMTs. The $\bar{\nu}_{e}$ from the reactor interacts with the detector via the inverse beta decay(IBD), and a positron together with a neutron come out after the interaction, namely, $\bar{\nu}_{e}+p \rightarrow e^{+}+n$. With the positron kinetic energy deposited followed by its annihilation, this event presents as a prompt signal. The neutron is captured by the Gd nucleus, and several gammas with total energy about $8 \mathrm{MeV}$ are emmited. This event forms the

This is an Open Access article published by World Scientific Publishing Company. It is distributed under the terms of the Creative Commons Attribution 3.0 (CC-BY) License. Further distribution of this work is permitted, provided the original work is properly cited. 
delayed signal. IBD candidates are selected through time-correlation method. The prompt energy from the positron gives an estimate of the incident $\bar{\nu}_{e}$ energy through: $E_{\nu} \simeq E_{e^{+}}+0.8 \mathrm{MeV}$.

\section{Energy Response Process}

The Daya Bay detector energy response can be understood as follows. A particle with its true energy deposit its energy in the $\mathrm{AD}$. For a $e^{-}, E_{\text {true }}$ is the kinetic energy; for a positron, $E_{\text {true }}$ is the sum of the kinetic energy and the energy from annihilation. After that, the LS translates the deposit energy into visible energy $E_{v i s}$. The visible photons are detected by photomultiplier tubes(PMT). After the calibration and reconstruction, $E_{v i s}$ is converted to be the reconstructed energy $E_{\text {rec }}$. The energy response is not linear due to scintillator and electronics effects. The non-linearity, $E_{\text {rec }} / E_{\text {true }}$, can be separated into two parts: the electronics nonlinearity, $E_{r e c} / E_{v i s}$, and the scintillator non-linearity, $E_{v i s} / E_{\text {true }}$. Non-linearity study is significant to deduce the true energy of positron from the reconstructed energy.

\section{Non-Linearity from the Electronics}

The electronics non-linearity is due to the interplay between slow component of LS light emitting $>100$ ns later after the first light(see Fig. 1) and the front end electronics system. Later hits formed by slow component may not be included in the hit collection, thus would make the collection efficiency decrease with increasing $E_{v i s}$. We used several models to parameterize the electronics non-linearity, for example, $\frac{E_{v i s}}{E_{r e c}}=\left(1-\alpha e^{\frac{E_{r e c}}{\tau}}\right)$.

\section{Non-Linearity from Liquid Scintillator}

The scintillator nonlinearity is particle and energy-dependent, and it is related to the intrinsic scintillator quenching and Cherenkov light emission. Different models are

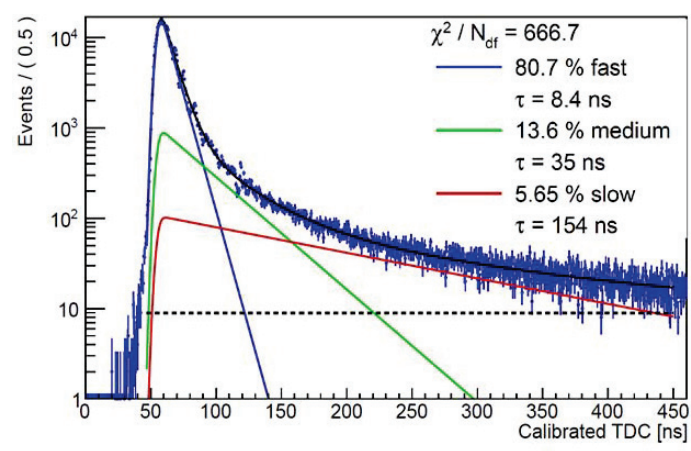

Fig. 1. Fast and slow components of LS photons, fitted by exponential functions. 
used to constrain the LS non-linearity of electron. One is a model using the Birk's law ${ }^{6}$ and Cherenkov radiation theory, $E_{\text {vis }} / E_{\text {true }}=f_{q}\left(E_{\text {true }} ; K_{B}\right)+K_{C} f_{c}\left(E_{\text {true }}\right)$, with the 1st term for quenching effect using the Birk's law, and the 2ns term for Cherenkov radiation. Another one is an empirical model with 4 parameters, $E_{\text {vis }} / E_{\text {true }}=\left(p_{0}+p_{3} E_{\text {true }}\right) /\left(1+p_{1} e^{-p_{2} E_{\text {true }}}\right)$. The gamma non-linearity and the electron non-linearity in the LS are connected by the energy conversion processes, gamma-rays interact with matter mainly in three ways, namely, Computon scattering,Photoelectric, and pair production, all these finally result in $e^{+}$or $e^{-}$. With a Geant4 simulation, the gamma to $e^{+} / e^{-}$converting probability function can be obtained. The non-linearity of gamma can be deduced from the secondary electron's non-linearity(see Fig. 2).

\section{Available Data to Constrain the Non-Linearity}

During a special calibration period in summer 2012, we used gamma sources and neutron sources deployed at the center of detector to do the nonlinearity study. Gamma sources such as ${ }^{137} \mathrm{Cs},{ }^{54} \mathrm{Mn},{ }^{40} \mathrm{~K}$, and neutron sources such as ${ }^{241} \mathrm{Am}-{ }^{9} \mathrm{Be}$

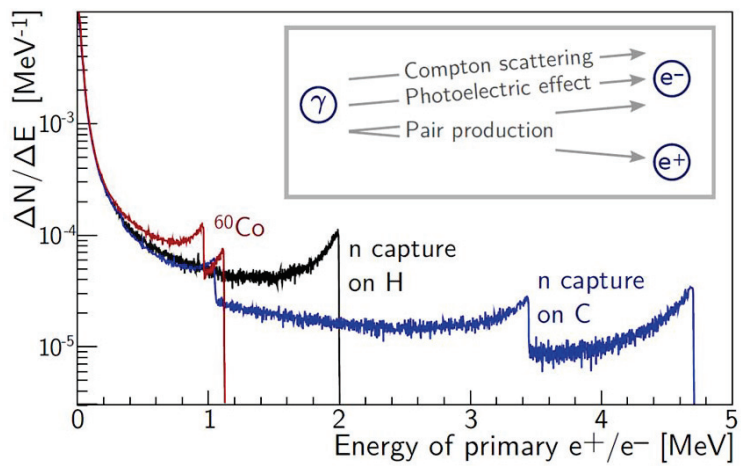

Fig. 2. The gamma to electron conversion probability density function via Geant4 for different gammas.
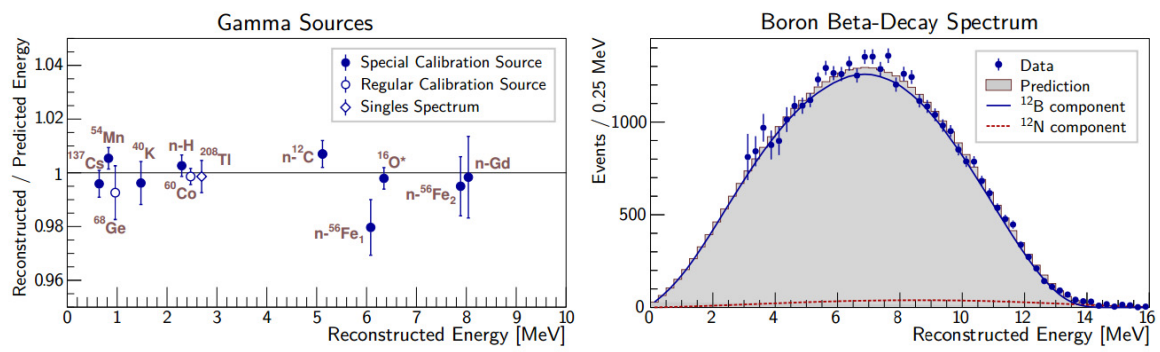

Fig. 3. The comparison of predicted vs measure gamma peaks(left) and ${ }^{12} B$ spectrum (right). 


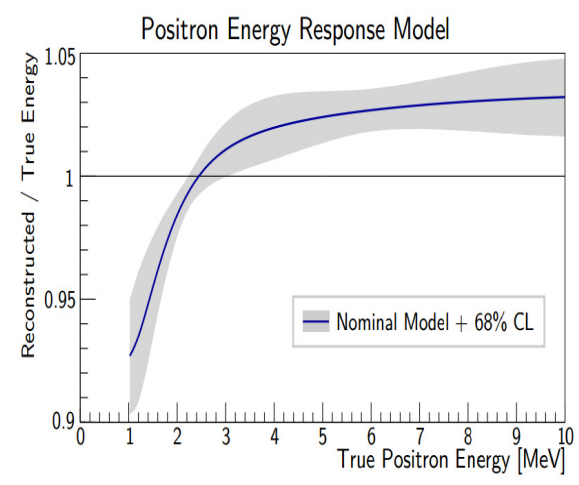

Fig. 4. Positron non-linearity result and its uncertainty contour.

and $P u-{ }^{13} C$ were used. Neutrons emitted from the neutron radiation sources can be captured on hydrogen or $\mathrm{Gd}$. A single $2.2 \mathrm{MeV}$ gamma is emitted when captured on hydrogen, providing one more gamma peak for nonlinearity study. The calibration data were fitted with the models described above, and consistency is obtained among different models. Energy spectrum from cosmic muon induced $\gamma+{ }^{12} B$ spectrum, is also used to test the consistency of the nonlinearity. The comparisons of the predicted using the best fit nonlinearity model vs measured gamma peaks and ${ }^{12} B$ spectrum are shown in Fig. 3.

\section{Summary}

After comparison between different models and comparison between models and data, the energy non-linearity for positron is show as follow(see Fig. 4), the shadow area represents the nonlinearity uncertainty both systematic and statistic combined within 1 sigma significance level, and the relative uncertainty of the non-linearity for positron is about $1.5 \%$.

\section{Acknowledgments}

We'd like to thank the PIC organization committee for this opportunity to present the nonlinearity studies at Daya Bay. We would also like to thank everyone who helped us on this contribution.

\section{References}

1. Daya Bay Collab. (F. P. An et al.), Chin.Phys.C. 108, 011001 (2013).

2. Spectral measurement. at Daya Bay (Daya Bay Collab.) Phys. Rev. Lett. 112, 061801 (2014).

3. B. Pontecorvo, Sov. Phys. JETP 6, 429 (1957) and 26, 984 (1968)

4. Z. Maki, M .Nakagawa and S. Sakata, Prog. Theor. Phys. 28, 870 (1962)

5. Daya Bay Collab. (F. P. An et al.), Phys. Rev. Lett. 108, 171803 (2012).

6. J. B. Birks, Phys. Lett. B 218, 365(1989) 